\title{
Impact of intensified pharmaceutical care on health related quality of life in patients with stroke in a tertiary care hospital
}

\author{
Dilip Chandrasekhar*, Anuja Pradeep, Asha Susan Geoji, Athira Elezebath George, Athira V., \\ Geethu k. Thomas \\ Professor and Head, Dept of Pharmacy Practice, Alshifa College of Pharmacy, Poonthavanam P.O, Perinthalmanna, Kerala, 679325, India
}

\section{A R T I C L E I N F O}

\section{Keywords:}

Adherence

Health related quality of life

Pharmaceutical care

Short form 36

Stroke

\begin{abstract}
A B S T R A C T
Background: The study investigates the pharmaceutical care concept to optimize drug therapy, minimize drug related problems and thereby improving health related quality of life (HRQoL) of stroke patients. Since stroke is a major health problem that negatively affects the patient's quality of life, it is relevant to the current pharmaceutical care scenario in a developing country like India. HRQoL related to stroke and life satisfaction after stroke is the important healthcare measures.

Methodology: Prospective interventional study conducted among inpatients of neurology department in a tertiary care referral hospital. 128 Patients with TIA/ischemic stroke were allocated to intervention group and control group (64 in each group). Pharmaceutical care was provided to the patients in the intervention group. SF-36 questionnaire was administered to both control group and interventional group to evaluate the patient's HRQoL. Adherence to entire therapy and antithrombotic therapy in both control group and intervention group were assessed using Morisky Medication Adherence Scales MMAS-8.

Results: Prevalence of ischemic stroke was more compared to hemorrhagic stroke. During the study, the intervention group showed high adherence to overall therapy than the control group. With an intensified pharmaceutical care, improvement in the HRQoL was observed in intervention group and was manifested in subscales and summary measures of SF-36.

Conclusion: The study enables demonstration and application of a pharmaceutical care program among stroke patients to prevent complications to improve HRQoL. This study indicates that pharmaceutical care can be performed in both hospital and community settings as it provides more benefit and positive impact on patient'HRQoL.
\end{abstract}

\section{Introduction}

Pharmaceutical care is the direct interaction between pharmacist and patient. In order to improve therapeutic compliance, a pharmacist promotes adequate follow-ups and provides counseling and thereby improves quality of life. Pharmaceutical care may be effective in reducing complications and in improving the quality of life of patients with chronic diseases, like stroke. Health related quality of life (HRQoL) related to stroke and life satisfaction after stroke are important healthcare outcomes. The main objective of our study was to investigate the impact of intensive pharmaceutical care (PC) versus standard care in a patient population with stroke. ${ }^{1}$

Stroke is defined by the World Health Organization (WHO) as 'a clinical syndrome consisting of rapidly developing clinical signs of focal disturbance of cerebral function lasting more than $24 \mathrm{~h}$ or leading to death with no apparent cause other than a vascular origin. ${ }^{2}$ Stroke can be either ischemic or hemorrhagic which can probably lead to disability in elderly. According to WHO, Quality of life is the individual's perception about their own position in their life in the context of culture, the value of where they live and related with their goals, expectation and standard. ${ }^{3}$ The effectiveness of an intervention can be evaluated by assessing patient's own opinion about their health. ${ }^{4}$ HRQoL assessment helps to determine and minimize the impact of risk factors that can lead to the development of rehabilitation strategy. ${ }^{5}$ Physical, functional, psychological, and social health are the 4 dimensions that can be used to assess the HRQOL. ${ }^{6}$ The Medical Outcomes of the patients were estimated using 36-item Short-Form Health Survey (SF-36). The SF-36 Health Survey contains 36 items that are scored out of eight scales: physical functioning (PF), role limitations due to physical health problems (RP), bodily pain (BP), general health (GH), vitality (VT), social

\footnotetext{
* Corresponding author at: Professor and Head, Dept of Pharmacy Practice, Alshifa College of Pharmacy, Poonthavanam P.O, Perinthalmanna, Kerala, 679325, India

E-mail address: dilipc@alshifacollegeofpharmacy.ac.in (D. Chandrasekhar).
} 
functioning (SF), role limitations due to emotional problems (RE) and mental health $(\mathrm{MH})$ and 2 summary scores. ${ }^{7,}{ }^{8}$ Short form 36 is a widely and frequently used, generic measure of health status. ${ }^{9}$

Pharmaceutical care (PC) is the provision of drug therapy by a responsible pharmacist for the purpose of achieving a definite outcome to improve the patients' quality of life. ${ }^{10} \mathrm{PC}$ ameliorates the patient's quality of life by optimizing drug therapy, minimizing drug-related problems, and improving self-management among patients. It can directly influence the HRQoL of patients.

Stroke, resulting to physical disability and even death, is a huge burden to patients in a developing country like India. ${ }^{11}$ Thus our main objective of the study was to evaluate the impact of PC on the HRQoL, determined by Short Form 36 (SF-36) among patients after stroke in the hospital.

\section{Methodology}

The study was a prospective interventional study conducted for a period of 1 year. The inpatients of neurology department in a private tertiary care referral hospital in Malabar region of Kerala were the research subjects. The study protocols were approved by the ethical and research committee of the hospital and was registered under Institutional Ethics Committee. The study purpose was explained and informed consent was obtained before enrolling study participants. Sample size calculations were done to improve the patients HRQoL after stroke on the basis of PC demonstrated that 60 patients were needed for each group. Since stroke has being commonly found in Malabar region of kerala due to metabolic syndromes mostly present in this region, a total of 140 patients were selected for the study. 8 patients were not meeting inclusion criteria and 4 of them were not willing to participate in the study. 128 patients were enrolled into intervention group $(n=64)$ and control group $(n=64)$ and were followed. Patients with TIA/ischemic stroke confirmed by CT/MRI scanning, Patients with previous history of stroke, Patients having no problem in their neuromuscular skeletal, psychiatric conditions, medical problem and other chronic illness such as metastatic cancer, AIDS, Patients without dementia or other cognitive impairment were included in our study. Patients with intracranial hemorrhage, seizure, migranious aura, patients with Barthel index of less than 30 points and patients unwilling to participate were excluded in the study. Diagnosis of stroke was established by clinical or CT findings by a consultant neurologist in the hospital. Patient demographic data was ascertained by interviewing each patient through medical records. Short form (SF)-36 was used to assess medical outcome. The SF-36 was suitable for self-administration, computerized administration, or administration by a trained interviewer in person or by telephone.

In phase I of the study, 128 patients with TIA/ischemic stroke were assigned to intervention group (IG) and control group (CG). SF-36 questionnaire was used to assess HRQoL at baseline and 30th day from the baseline.

In phase II Pharmaceutical care (PC) was provided to the patients in the intervention group. The main intervention was done in case of educating patients and proving rational drug therapy to them. Counseling was provided realted to the causes of stroke, risk factors and symptoms such as hypertension, diabetes mellitus, hyperlipidemia, and atrial fibrillation as well as secondary prevention. Counseling in current medications, effects, dosage, side effects, drugs for secondary prevention, and cardiovascular risk factors by using 'discharge medication letter' during their discharge was also provided. The patients were explained about common misconceptions about therapy and various benefits of adhering to medication therapy. This helped patients to reschedule new drug regimens prescribed.

In phase III, on the 30th day from baseline, the SF-36 questionnaires were repeated to both CG and IG. The SF-36 questionnaires from two phases were compared. Two types of SF-36 scores were generated: 8 subscales scores and two summary measure scores. Based on these scores the impact of pharmaceutical care on HRQOL was evaluated. After 30 days, comparison of adherence to entire therapy and adherence to antithrombotic therapy in both control group and intervention group were assessed using Morisky Medication Adherence Scales (MMAS-8). After intervention patient's knowledge in various aspects like: Medication Regimen and Patient Knowledge in medication name and directions were assesed in both control group and intervention group. Data collected from the study was tabulated in Microsoft Excel 2010 and were keyed into the Statistical Package for Social Sciences (SPSS Inc., Chicago, IL, USA) computer software version 20 for windows and analyzed by appropriate statistical methods. Statistical analysis was both descriptive at $95 \%$ confidence level (CI). The students' $t$-test was used to compare the means of both groups. A twotailed probability value of $<0.05$ was considered to be statistically significant.

\section{Results}

All 128 patients (intervention group $=64$, control group $=64$ ) completed the entire study and were followed for a period of one year. The study investigated the impact of intensive pharmaceutical care versus standard care in a patient population with stroke. Patient's baseline characteristics are summarized in Table1. There were no significant differences with regards to age $(\mathrm{p}=0.864)$ and marital status $(p=0.49)$ between intervention group and control group. Gender distribution of stroke $(\mathrm{p}=0.003)$ and food habits $(\mathrm{p}=0.002)$ showed a significant difference between both groups. In our study, patients following non vegetarian diets were mostly observed to develop stroke. Study results revealed that about $93 \%$ of subjects were non-vegetarian and $7 \%$ were vegetarian. Clinical characteristics of the study population are summarized in Table 2. About $51 \%(n=65)$ of reported cases were with right side brain lesion and $45.31 \%(n=58)$ cases were with left hemisphere lesion. There was no statistically significant difference ( $p=0.57$ ) between control group and intervention group for the occurrence of brain lesion. Study results showed that, patients with hypertension developed stroke at a faster rate in both control and intervention group. In control group $18.8 \%$ of the patients had previous history of stroke and $20.3 \%$ of the patients in intervention group. In

Table 1

Baseline characteristics of study population.

\begin{tabular}{|c|c|c|c|}
\hline CHARACTERISTICS & \multicolumn{2}{|c|}{$\%$ OF TOTAL PATIENTS } & P VALUE \\
\hline \multicolumn{3}{|l|}{ 1.AGE } & \multirow[t]{3}{*}{0.582} \\
\hline Minimum & $3.125 \%$ & & \\
\hline Maximum & $38.28 \%$ & & \\
\hline \multicolumn{3}{|l|}{ 2.GENDER } & \multirow[t]{3}{*}{0.003} \\
\hline Male & $63.3 \%$ & & \\
\hline Female & $36.7 \%$ & & \\
\hline \multirow[t]{2}{*}{ 3.MARITAL STATUS } & INTERVENTION & CONTROL & \multirow[t]{4}{*}{0.49} \\
\hline & GROUP & GROUP & \\
\hline Married & $84.4 \%$ & $79.7 \%$ & \\
\hline Widowed & $15.6 \%$ & $20.3 \%$ & \\
\hline \multicolumn{3}{|l|}{ 4.HEALTH INSURANCE } & \multirow[t]{3}{*}{0.009} \\
\hline Yes & $28.1 \%$ & $42.2 \%$ & \\
\hline No & $71.9 \%$ & $57.8 \%$ & \\
\hline \multicolumn{3}{|l|}{ 5. WAY OF LIVING } & \multirow[t]{3}{*}{0.19} \\
\hline With spouse & $57.8 \%$ & $68.8 \%$ & \\
\hline With others & $42.2 \%$ & $31.3 \%$ & \\
\hline \multicolumn{3}{|l|}{ 6. QUALIFICATION } & \multirow[t]{4}{*}{0.001} \\
\hline Elementary school & $45.3 \%$ & $40.6 \%$ & \\
\hline Higher secondary & $39.1 \%$ & $31.3 \%$ & \\
\hline Undergraduate degree & $15.6 \%$ & $28.1 \%$ & \\
\hline \multicolumn{3}{|c|}{ 7. RESIDING NEIGHBOURHOOD } & \multirow[t]{3}{*}{0.68} \\
\hline Urban & $23.4 \%$ & $26.6 \%$ & \\
\hline Village & $76.6 \%$ & $73.4 \%$ & \\
\hline
\end{tabular}


Table 2

Clinical characteristics of study population.

\begin{tabular}{lclc}
\hline & $\begin{array}{l}\text { INTERVENTION } \\
\text { GROUP }\end{array}$ & $\begin{array}{l}\text { CONTROL } \\
\text { GROUP }\end{array}$ & P VALUE \\
\hline $\begin{array}{l}\text { 1.TYPE OF STROKE } \\
\text { Ischemic stroke }\end{array}$ & $78.1 \%$ & $70.3 \%$ & 0.44 \\
$\begin{array}{l}\text { Hemorrhagic } \\
\text { Transient ischemic }\end{array}$ & $15.6 \%$ & $17.2 \%$ & \\
$\quad$ stroke & $6.3 \%$ & $12.5 \%$ & \\
2. SIDE OF BRAIN LESION & & \\
Right hemisphere & $54.7 \%$ & & \\
Left hemisphere & $40.6 \%$ & $46.9 \%$ & \\
Cerebellar & $4.7 \%$ & $50.0 \%$ & 0.63 \\
3. NUMBER OF COMORBIDITIES & $3.1 \%$ & \\
Nil & $14.1 \%$ & & \\
2 & $26.6 \%$ & $18.8 \%$ & \\
$>3$ & $6.3 \%$ & $23.4 \%$ & \\
\hline
\end{tabular}

control group (10.9\%) and intervention group (6.20\%), patients had a previous history of diabetes, dyslipidemia and hypertension.

Educational interventions like Counseling about administration of drugs, use of drug, lifestyle modifications, heath problem, and actual or potential adverse drug reaction were provided to the entire study population. This helped to re-schedule new drug regimen in 55 patients. Adverse drug reactions in 27 patients were resolved. The adherence rate of total population has been described in Table 3. The overall adherence to the entire drug therapy has increased in intervention group after the pharmaceutical care. The difference was found to be extremely significant on analysis using Chi-square test with $\mathrm{p}=0.001$ (X2 = 39.18). About $59.4 \%$ of patients had exhibited high adherence to drug therapy in intervention group and 9.4\% in control group. 56.3\% patients showed low adherence rate of therapy in control group and $14.1 \%$ in the intervention group. The overall adherence to the anticoagulant therapy increased in the intervention group after the counseling. The difference was found to be extremely significant on analysis using Chi-square test with $\mathrm{p}=0.001(\mathrm{X} 2=36.01) .64 \%$ of patients in the intervention group exhibited high adherence to anti-coagulant therapy and $13 \%$ in control group. Headache (8 cases), gastritis (5 cases), and rashes ( 4 cases) were the most observed ADRs in intervention group during the study period. The HRQoL assessment in IG and CG using SF36 at baseline and after 30 days has been explained. Fig. 1 shows score distribution of the SF-36 (8 scales) in control group at baseline and after 30 days. Fig. 2 shows score distribution of the SF-36 (8 scales) at baselines of intervention \& control group. Fig. 3 shows score distribution of the SF-36 (8 scales) at 30th day of control group \& intervention group.

The analysis of quality of life by HRQoL parameter in CG deteriorated significantly in 6 subscales out of 8 over time. The subscales Role limitations were physical (RP), General Medical Health (GH), vitality (VT), Social functioning(SF), Role Limitation-Emotional(RE), Mental Health $(\mathrm{MH})$ decreased significantly. Upon admission patients of both, IG and CG, showed no significant differences in the 8 subscales. The score distribution after 30 days revealed that the subscales RP, GH, VT,

Table 3

Adherence rate in Intervention group and control group to therapeutic regimen.

\begin{tabular}{lcll}
\hline & Intervention group & Control group & P value \\
\hline $\begin{array}{l}\text { 1. Adherence to entire medication regimen } \\
\text { High adherence }\end{array}$ & $59.4 \%$ & $56.3 \%$ & \\
Medium adherence & $26.6 \%$ & $34.4 \%$ & 0.0008 \\
Low adherence & $14.1 \%$ & $9.4 \%$ & \\
2. Adherence to Anticoagulant therapy & & \\
High adherence & $64 \%$ & $13 \%$ & 0.0009 \\
Medium adherence & $71 \%$ & $30 \%$ & \\
Low adherence & $6 \%$ & $16 \%$ & \\
\hline
\end{tabular}

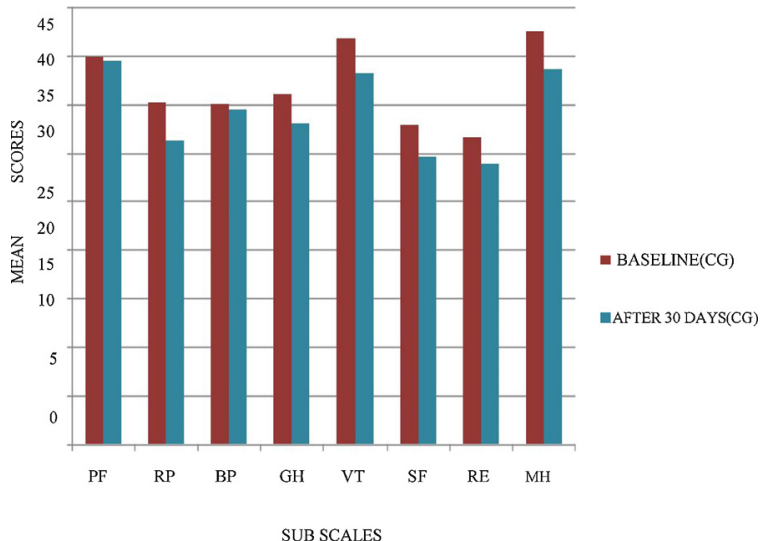

Fig. 1. Score distribution of the SF-36 (8 scales) in control group at baseline and after 30 days. HRQoL parameter of patients in CG reduced significantly in 6 of the 8 subscales over time. The subscales Role limitations - physical (RP), General Medical Health (GH), vitality (VT).Social functioning (SF), Role Limitation-Emotional (RE), Mental Health (MH) decreased significantly.

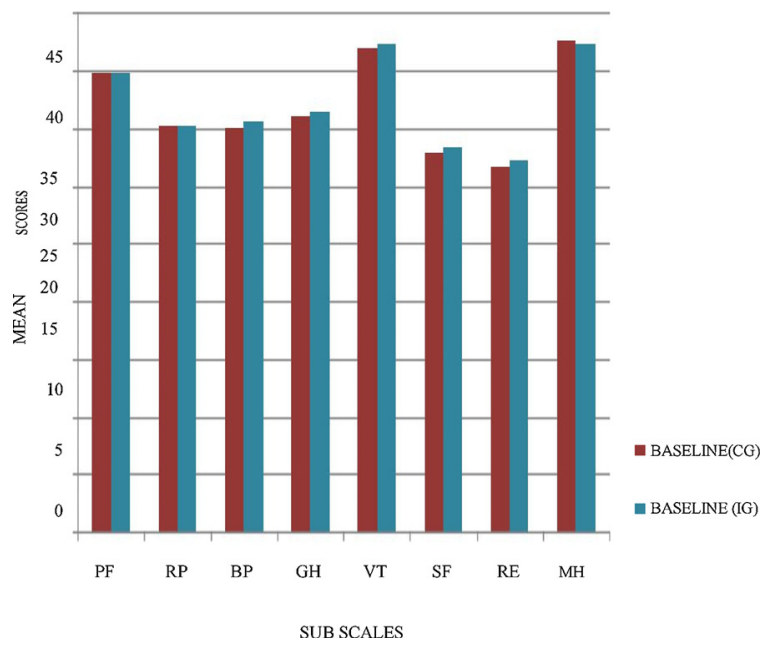

Fig. 2. Score distribution of the SF-36 (8 scales) at baselines of intervention and control group. Patients in IG and CG, showed no significant differences at baseline period for all the 8 subscales in SF 36 Questionnaire.

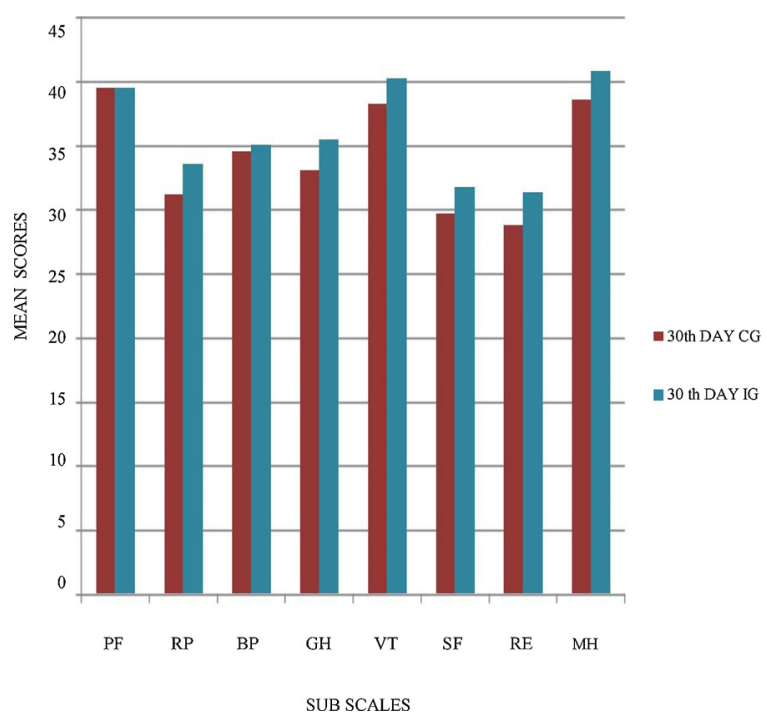

Fig. 3. Score distribution of the SF-36 (8 scales) 30th day scores of control group \& intervention group. After 30 days the subscales RP, GH, VT, SF, RE, MH scores were significantly higher in IG than in CG. 
SF, RE, MH score were significantly higher in IG patients than in CG patients. There were no significant differences in HRQoL between the groups on their follow ups. For the CG, a significant decline in (Physical component) PCS and (Mental component) MCS was observed between baseline and on follow-up (PCS: $p=0.001$; MCS: $p=0.001$ ). Changes in PCS and MCS between study entry and follow-ups in IG were not statistically significant. The correlation between number of disease and quality of life were analyzed. At baseline, study revealed a trend of poor QOL in patients who had co-morbid conditions $(\mathrm{p}<0.001)$ (Pearson correlation coefficient $=0.838 ; \mathrm{R} 2=0.702$ ). Scatter plot was used to demonstrate the correlation.

\section{Discussion}

The overall HRQoL in stroke patients was significantly improved in 6-12 months in intervention group when compared with control group. The pharmaceutical care intervention impacted positively on patient's HRQoL which was both clinically and statistically significant. This also improved adherence to lifestyle advices. The results of this intervention were similar to that of a prospective intervention study conducted by Hohmann et al. ${ }^{1}$ in a major hospital in Germany for a period of 12 months. As per the inclusion and exclusion criteria, a total of 128 subjects (64 in each phase) were enrolled in our study on the basis of convenience sampling. Age showed an important risk factor contributing to stroke. A similar pattern was observed in study conducted by Nuray Dayapoglu et al. ${ }^{12}$ In our study, patients with ischemic stroke were more prevalent than hemorrhagic stroke. A study conducted by Franke et al. ${ }^{13}$ shows prevalence of ischemic stroke was more compared to hemorrhagic stroke in both control $(70.3 \%)$ and intervention group (78.1\%). It has been suggested that stroke lesion location can be of paramount importance for the patient's HRQoL in a study conducted by de Haan et al. ${ }^{14}$ In this study the right hemisphere of the brain was mostly affected in about $54 \%$ of the patients which is consistent with the results by Nuray dayapoglu et al. ${ }^{12}$ In this study the most frequent cardiovascular risk factor in both groups was hypertension (78\%), followed by diabetes (45\%), combination of diabetes and hyperlipidemia (27.34\%), history of cardiac problems (20.31\%). A similar multi-center prospective observational study conducted by Vilai Kuptniratsaikul et al. ${ }^{15}$ in Thailand concludes that the complication after stroke was common problems causing lose of self care functions, psychological impact, social disability and decreased quality of life. The major underlying medical diseases were hypertension $(75.7 \%)$ followed by dyslipidemia (55.1\%), diabetes mellitus (29\%), cardiac diseases (18.7\%) and previous stroke (15\%).

A systematic review and meta analysis study conducted by Mohammed et al. concludes that the development of a suitable HRQoL measure for pharmaceutical interventions may help to generate better evidence for the contribution of pharmacist services to improve HRQoL. ${ }^{16}$

Stroke patients imposed the greatest HRQoL deficit among all other co-morbidities in both IG and CG. The HRQoL deficit associated with stroke and hypertension has been reported by Ekwunife et al. ${ }^{17}$ Antihypertensive drug induced Headache and antiplateletic drug induced gastritis, were the most adverse reactions observed in intervention group during the study period which showed a consistent pattern in study conducted by Gholami et al and Singhal Rohit et al. ${ }^{18}$ Patients with more than one diseases and with polypharmacy had poor quality of life which showed a similar result in study conducted by Divaldo pereira et al. ${ }^{19}$ about pharmaceutical care in elderly, in which there was a positive correlation between number of diseases and number of comorbidities with QOL.

This study concludes with a positive impact on pharmaceutical care provided by the pharmacist in improving HRQoL in patients with stroke which showed a similar result in a study conducted by Hohmann et al. ${ }^{1}$ Their findings indicate that an intensified education and care of patients after stroke by dedicated pharmacists based on a concept of PC may have a positive impact on HRQoL.

\section{Conclusion}

The study concludes that pharmaceutical care performed in the hospital settings was feasible and had a clear benefit and positive impact on patient's HRQoL. Moreover, it was a step towards monitoring and implementation of pharmaceutical care within hospitals and the community setting, for evaluating the role of pharmacists within a specific therapeutic team. It was found that due to an intensified involvement by pharmacist a pharmaceutical care concept had an ability to reduce drug related problems, improved patient's satisfaction and stabilized the HRQoL in several scales. Sedentary life style, with increasing age was more prone for stroke especially hemorrhagic stroke over the ischemic one. The intensified pharmaceutical care given to intervention group resulted in a greater improvement in the HRQoL subscales and summary scores. There are several studies which can demonstrate that PC may have a positive impact on the patients HRQoL. The major highlights of the study results is about the pharmacist's role is in improving the appropriateness of prescription, prevention of medication related problems, improving patient knowledge about disease and drugs. These results in the improvement of medication adherence and thereby improving patient outcomes. The study enables demonstration and application of a pharmaceutical care program among stroke patients to prevent complications to improve HRQoL. We adopted certain strategies to strengthen pharmaceutical care for the improvement patients quality of life.

\subsection{Limitations of the study}

The limitations of the present study include the fact that no blinding or randomization could be performed. Sample size was small. Lack of information on socioeconomic status, financial resources or caregiver support in patients of either group, which could have influenced the outcome of the patients.

\section{References}

1. Hohmann Carina, Radziwill Roland, Klotz Juergen M, Jacobs Andreas H. Health related quality of life after ischemic stroke: the impact of pharmaceutical interventions on drug therapy. Health Qual Life Outcomes. 2010;8(59).

2. Tyrrell Pippa, Rudd Anthony, Cullen Katherine, Richards Alison, Swain Sharon, Turner Claire. National clinical guideline for diagnosis and initial management of acute stroke and transien ischemic attack. London: Wiley; 2008:432.

3. Azman AB, Sararaks S, Rugayah B, Low LL, Azian AA, Geeta S, et al. Quality of life of the Malaysian general population, result from a postal survey using SF- 36. Med J Malays. 2003;58:694-711.

4. Kuroda A, Kanda T, Sakai F. Gender differences in health related quality of life among stroke patients. Geriatr Gerontol Int. 2006:165-168.

5. Liu WJ, Chew TF, Chiu ASF, Zaki M. Quality of life of dialysis patients in Malaysia. Med J Malays. 2006(61):540-546.

6. Carod-Artal J, Egido JA, González JL, de Seijas EV. Quality of life among stroke survivors evaluated 1 year after stroke: experience of a stroke unit. Stroke. 2000:31:2995-3000.

7. de Haan R. Measuring quality of life after stroke using the SF-36. Stroke. 2002;33:1176-1177.

8. Madden S, Hopman WM, Bagg S, Verner J, Callaghan CJ. Functional status and health related quality of life during inpatient stroke rehabilitation. Am J Phys Med Rehabil. 2006;85:831-838.

9. Hobart JC, Williams LS, Moran K, Thompson AJ. Quality of life measurement after stroke: uses and abuses of the SF-36. Stroke, 2002:33:1348-1356.

10. Anderson C, Laubscher S, Burns R. Validation of the short form 36(SF-36) health survey questionnaire among stroke patients. Stroke. 1996(27):1812-1816.

11. Durai Pandian Jeyraj, Sudhan Paulin. Stroke epidemiology and stroke care services in India. Journal Stroke. 2013;15(3):128-134.

12. Dayapoglu Nuray, Tan Mehtap. Quality of life in stroke patients. Neurol India. 2010(58):697-701

13. Franke CL, Van Swieten JC, Algra A, Van Gijn J. Prognostic factors in patients with intracerebral hematoma. J Neurol Neurosurg Psychiatry. 1992(27):40-46.

14. Haan RJ, Limburg M, der Meulen V, Jacobs HM, Aaronson NK. Impact of stroke type and lesion location. Stroke. 2006:402-408.

15. Kuptniratsaikul Vilai, Kovindha Apichana, Suethanapornkul Sumalee, Manimmanakorn Nuttaset, Archongka Yingsumal. Long-term morbidities in stroke survivors: a prospective multicenter study of Thai stroke rehabilitation registry. 
Stroke. 2013(78):345-352

16. Mohammed Mohammed A, Moles Rebekah J, Chen Thimothy F. Impact of pharmaceutical care intervention on HRQoL outcomes. Ann Pharm. 2016;50:862-881.

17. Ekwunife OI, Aguwa C, Adibe M, et al. Health state utilities of a population of Nigerian hypertensive patients. BMC Res Notes. 2011;4:528.

18. Rohit Singhal, Khaleel Ahmed, Santai DD. Reporting and monitoring of adverse drug reactions with cardiac drugs. IRJP. 2011(7):116-119.

19. Pereira de Lyra Divaldo, et al. Impact of pharmaceutical care interventions in the identification and resolution of drug-related problems and on quality of life in a group of elderly outpatients in Ribeirao Preto (SP), Brazil. Ther Clin Risk Manag. 2007(6):989-998. 